\title{
Colchicine trial in PFAPA Syndrome and MEFV-negative patients
}

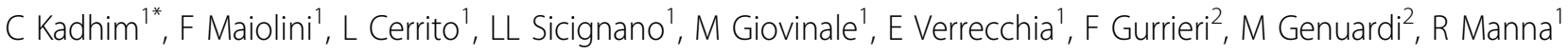 \\ From 8th International Congress of Familial Mediterranean Fever and Systemic Autoinflammatory Diseases \\ Dresden, Germany. 30 September - 3 October 2015
}

\section{Introduction}

PFAPA Syndrome (Periodic Fever, Aphthous stomatitis, Pharingitis, and cervical Adenitis) is the most common periodic fever in childhood; the diagnosis is based on clinical criteria. Familiar Mediterranean Fever (FMF) is a monogenic autosomal recessive autoinflammatory disease, whose diagnosis is based on clinical elements, supported by MEFV genetic mutations. When there is only a mutation or no one, the patient undergoes a trial with colchicine for 4-6 months, and diagnosis is confirmed in case of clinical response and fever early recurrence after suspension. Current treatment of PFAPA is symptomatic. Febrile episodes show a rapid response to the administration of one or two doses of prednisone $(1-2 \mathrm{mg} / \mathrm{kg})$ or betamethasone (0.1-0.2 mg/kg). Total requirement of steroid increases over time, and the frequency of attacks worsens the quality of life of patients. In literature, the prophylaxis of PFAPA febrile attacks with colchicine (0.5-1 $\mathrm{mg} /$ day) has been tested only on a few patients, with controversial results.

\section{Objectives}

Considering the similarities between FMF MEFV-negative patients (MEFVneg) and PFAPA patients, we aimed to demonstrate that colchicine is effective in PFAPA too: positive response was evaluated in terms of reduction in frequency $>50 \%$ and severity of attacks $>50 \%$.

\section{Materials and methods}

We conducted a prospective cohort study (from September 2012, still ongoing), comparing two groups: 67 MEFVneg and 51 PFAPA patients. 36 of the latter group underwent colchicine trial, after obtaining informed consent.

\section{Results}

We assessed the response of PFAPA patients to colchicine preventive treatment: good response was observed in $75 \%$ (27 patients of 36 ), and a non-response in $25 \%$ (9 pts). The effective treatment rate of MEFVneg is $100 \%$, by definition. The average dose of colchicine administered in PFAPA was $1.14 \mathrm{mg} /$ day, compared to MEFVneg (1.34 mg/day). The dose per kilogram of body weight is $0.020 \mathrm{mg} / \mathrm{kg} /$ day in both groups. We can state that the colchicine dose requirement in PFAPA coincides to the one of FMF patients.

\section{Conclusion}

Our study showed that colchicine regimen is effective in $75 \%$ of cases. Prophylaxis with colchicine should be offered to all PFAPA patients, instead of steroids or other symptomatic therapy (as paracetamol or ibuprofen), before the treatment with anti-IL1 $\beta$ biologic drugs, with considerable savings in pharmacoeconomics.

\section{Authors' details \\ ${ }^{1}$ Catholic University of the Sacred Heart, Internal Medicine, Rome, Italy. \\ ${ }^{2}$ Catholic University of the Sacred Heart, Human Genetic, Rome, Italy.}

Published: 28 September 2015

doi:10.1186/1546-0096-13-S1-05

Cite this article as: Kadhim et al:: Colchicine trial in PFAPA Syndrome and MEFV-negative patients. Pediatric Rheumatology 2015 13(Suppl 1):O5.

${ }^{1}$ Catholic University of the Sacred Heart, Internal Medicine, Rome, Italy

Full list of author information is available at the end of the article 